\title{
Arcuate Fasciculus Lateralization is not a Marker of Language Lateralization in Children with Intractable Epilepsy
}

\author{
Byron Bernal ${ }^{1,2,3 *}$, Alfredo Ardila ${ }^{4}$, Monica Rosselli ${ }^{5}$, Michael Duchowny ${ }^{2}$, Magno R Guillen $^{1}$ and Nolan Altman ${ }^{1}$ \\ ${ }^{1}$ Department of Radiology, Nicklaus Children's Hospital, Miami, FL, USA \\ ${ }^{2}$ Brain Institute, Miami Children's Hospital, Miami, FL, USA \\ ${ }^{3}$ Herbert Werthein, College of Medicine, Pediatric Department, FIU, Fl, USA \\ ${ }^{4}$ Psychology Program, Albizu University, Miami, Florida, USA \\ ${ }^{5}$ Department of Psychology, Florida Atlantic University, Davie, FL, USA \\ *Corresponding Author: Byron Bernal, Department of Radiology, Nicklaus Children's Hospital, Miami, FL, USA.
}

Received: July 18, 2019; Published: September 13, 2019

DOI: 10.31080/ASNE.2019.02.0105

\begin{abstract}
Objective: The arcuate fasciculus (AF), is an asymmetric bundle that connects areas of comprehension and verbal expression. The aim of this study is to test the hypothesis that arcuate fasciculus dominance and language lateralization are correlated in a pediatric group with intractable epilepsy by utilizing DTI and language fMRI lateralization indices (LI).

Methods: 33 right-handed children presenting intractable epilepsy were retrospectively included. Language lateralization was ascertained with a controlled fMRI utilizing an Auditory Description Task paradigm and lateralization indices (LI) ascertained. Deterministic fiber tracking based on DTI-MRI was performed. LI and fiber number count for AF were compared Correlations were performed utilizing Pearson test. A correlation coefficient greater than 0.3 was accepted as correlation present. Anova test was also utilized to study confound variables.
\end{abstract}

Results: There was no significant correlation between AF lateralization and language lateralization for any domain $(R=-0.19$ for expressive; $R=0.03$ for receptive). 31 patients had either left (15) or bilateral (16) arcuate dominance. Two patients had discordant right sided AF dominance (6.1\%). In only 20 out of 33 cases (61\%) arcuate fasciculus fibers had a terminus in the left Broca's area (pars opercularis of the IFG).

Conclusions: The only correlation obtained was between the number of fibers in the right AF and the AF-LI.

Classification of evidence: This study provides class IV evidence that Arcuate Fasciculus Lateralization is not a marker of Language Lateralization.

Keywords: Language; Laterality; Arcuate Fasciculus; Brain Connectivity; Epilepsy Surgery

\section{Introduction}

Language lateralization is based on a hemispheric advantage arising from predetermined structural connectivity of functional networks sub serving language competence. Among all the associative tracts, the main candidate for the support of this functional advantage is the arcuate fasciculus. While the arcuate fasciculus is left-sided lateralized in right handed subjects [1] it is also lateralized to the left hemisphere in patients with proven right hemisphere language function [2,3].These findings are puzzling as intraoperative electrical stimulation of the AF produces phonological Paraphasia indicating a functional contribution [4].
It is of interest that reports of dissociated language lateralization have been always shown right sided functional language (by fMRI or Wada) compared to left sided AF lateralization. This asymmetry of the AF is not only macro-anatomical as AF asymmetries are also related to diffusion related variables [e.g] $[5,6]$.

Current DTI techniques for sedated subjects are widely available for AF tracking in any patient regardless of age or cognitive level. Nevertheless, the comparison of functional language lateralization with AF lateralization remains limited, and mostly studied in normal adult subjects; to our knowledge, similar investigations have not been performed in the pediatric population. 
The aim of this work is to present a quantitative correlation study between scores derived from a controlled-language f MRI mapping and an index of structural lateralization based on scalars derived from the AF in a group of pediatric patients with intractable epilepsy. We hypothesized that AF lateralization correlates with language lateralization and that an AF-fiber count lateralization index correlates with language lateralization indices based on voxel count. We further hypothesized that the end - point of the anterior terminus may predict expressive lateralization of language.

\section{Methods}

Participants

Demographic details are presented in table 1 . Thirty-three right-handed children and adolescent patients (age mean/SD = 15.8 / 2.4; 22 female) were selected, in a retrospective study from the pool of clinical fMRI mapping performed in our service, using the following selection criteria: (1) diagnosis of intractable epilepsy in candidates for neurosurgery, (2) acquisition of a controlled task-related fMRI for language, and (3) determination of the AF by a DTI sequence.

Exclusion criteria included: (1) prior neurosurgery, (2) left handedness, (3) implanted device that could be source of artifact or difficult co-registration, and (4) gross brain parenchymal asymmetry. Although not all cases had a standard Neuropsychologist evaluation, those who were tested, having verbal IQ score lower than 85 , or full scale IQ lower than 75 , were excluded. The study protocol was approved by the Western IRB.

We defined the AF, as that part of the superior longitudinal fasciculus formed by the curved-shaped bundle traveling from the posterior third of the superior and middle temporal gyri (posterior terminus) to a region encompassing the inferior third of the pre-central and the inferior frontal gyrus (anterior terminus).

\section{Data acquisition}

FMRI acquisition was performed using a Philips 1.5 Tesla Magnet, Ingenui (Netherlands). We utilized a 15-channel dS Sense enhanced parallel imaging head coil (Philips, Netherlands). Stimuli were delivered by monophonic bi-neural presentation via headphones fed by tubing from a ceramic transducer placed near the magnet (FMRI Consulting, Inc., USA). Responses were acquired via a fiber-optic wired button box device (FMRI Consulting, Inc., USA).

\section{Sequences}

All patients had a 3D anatomical series utilized for intrasubject co-registration. The series consisted of an axial T1-weighted 3D sequence; matrix xy dimensions 256 x 256 voxels, FOV $240 \mathrm{~mm}$.
Voxels were electronically re-dimensioned to 1 x 1 x $1 \mathrm{~mm}$. Likewise, all cases had an auditory description task (described below). For this purpose a BOLD-sensitive gradient echo-planar single shot sequence was obtained utilizing 150 timepoints of 21 interleaved axial cuts obtained with FOV: 240; Matrix 64 x 64; TR / TE / FA = 2000ms / 45 ms / 90 deg. Deterministic Diffusion Tensor Imaging (DTI) was also obtained in each case utilizing a single-shot spin-echo echo-planar sequence with the following settings: 65 axial straight slices, 15 directions; TR / TE / FA: 2944 ms / 85 ms / 90 degrees; single reference B value of $900 \mathrm{~s} / \mathrm{mm}^{2}$.

\section{FMRI paradigms}

All patients performed an Auditory Description Task in a block design of 5 ONs and 5 OFFs of 30 seconds each, starting in OFF. Each ON condition consisted of 7 sentences in English, delivered in binaural manner utilizing headphones and tubing connected to a ceramic speaker placed near the magnet and conveniently connected to a computer localized in the operators room. 3 - 4 of these sentences, per epoch, were true statements. The OFF condition consisted of pure tones of $600 \mathrm{~Hz}, 200 \mathrm{~ms}$ of duration, pseudo-randomly distributed amid gibberish. Patient responded by pressing a button: during $\mathrm{ON}$, when the sentence presented was TRUE; during OFF, for each tone (3 - 4 per epoch). The patient's motor responses were counterbalanced in hits between conditions. Gibberish was obtained by playing backwards the same sentences utilized during ON. A graphical explanation of the paradigm is shown in figure 1 . Patients were instructed to remain perfectly still, keep eyes open and blinking at will.

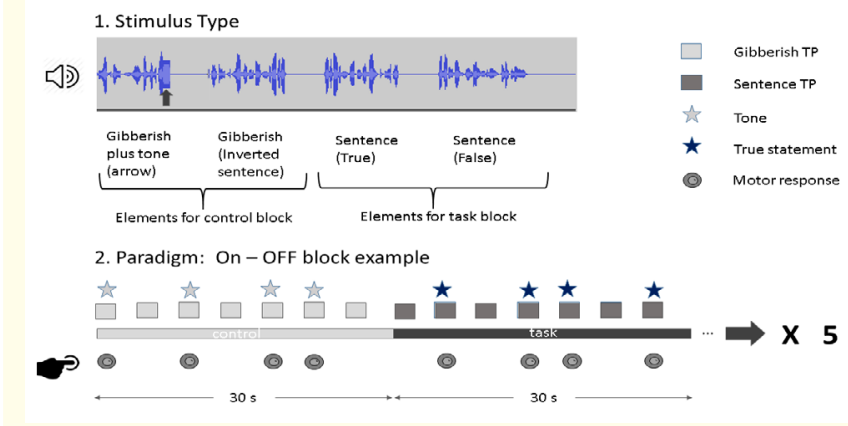

Figure 1 Paradigm illustration: ADT. Brief exemplification of the 4 types of auditory stimulus are provided. Gibberish was obtained inverting each of the statements used for the $\mathrm{ON}$ epoch. Notice the inverse signal profile between stimulus 2 (Gibberish) and 3 (True sentence). Hits are Tones (in off epochs) and true statements (in on epochs). Responses are counterbalanced across the paradigms. 5 repetitions of the cycle conforms the entire paradigm. 


\section{Post-processing Functional MRI}

All sequences were processed at the individual level. Initially, the BOLD sequence was re-aligned, high-band-pass filtered; smoothed with 7 × 7 × 7 mm kernel; motion-corrected and co-registered with the patient's 3D - T1 anatomical image. A General Linear Model was applied modeling the block design, utilizing FEAT from FSL (fMRI Expert Analysis Tool, version 5.98 from FSL 4.1 .9 (http:// www.fmrib.ox.ac.uk/). The main regressor was convoluted with double-gaussian and the hemodynamic response function models to account for the delay and ramping up and down of the BOLD function. Results were Thresholding at $\mathrm{P}<0.01$, corrected for multiple comparisons utilizing a cluster size threshold of 27 voxels. Results are finally presented in color maps overlaid on top of the 3D-T1 anatomical image (Figure 2).
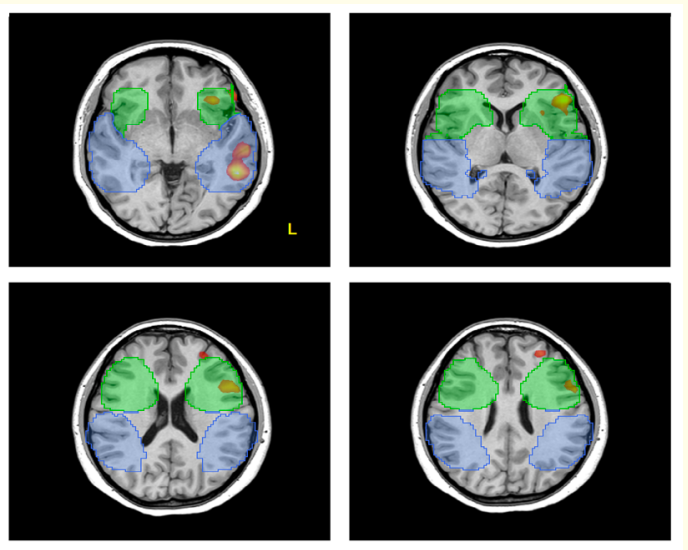

Figure 2 fMRI activation map (ADT): Selected axial cuts displaing the fMRI activation overlaid on an Axial T1 weighted 3D brain MRI. Images are oriented in radiological convention. 3D-ROIs for LI calculation are displayed in translucent colors, green for expressive, blue for receptive. Activation is shown in color from red (low intensity) to yellow (high intensity). Notice the exclusive lateralization of active voxels in the left hemisphere in a case with language $\mathrm{LI}=1$ (all voxels on the left side).

\section{DTI and tractography processing}

The DTI dataset was processed with the dTV II (diffusion Tensor Visualizer II) plugin available on the open-source software Volume - one [7]. The voxels were interpolated to isotropic dimensions. A standard table for 15 directions was applied to obtain color-coded directional, FA, and mean diffusivity maps. Manually definition of two ROIs was performed by the same author (BB) to selectively track the arcuate fasciculus (long segment of the SLF), in both si- des. The two ROIs were defined, linked with an AND Boolean operator: The first ROI, the coronal ROI was obtained by outlining an area found in the coronal view, just rostral to the splenium, identified as a green triangular-shaped tract lateral to the corticospinal tract; the second ROI, is placed in an axial view and was obtained by outlining an area found in an axial cut at the most inferior level of the splenium, identified as blue dots lateral to the green associative tracts on the temporo-occipital junction (Figure 3).

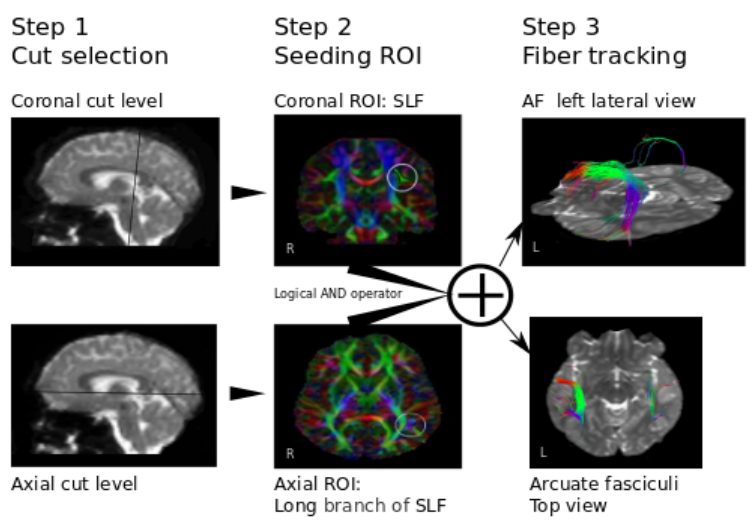

Figure 3 Arcuate fasciculus tracking method: First column shows a mid-sagittal B0 slice. The black line indicates the selected level of a coronal (upper) and an axial cut (lower) where the main two ROIs are localized for tracking of the Arcuate fasciculus. For the coronal selection, the plane is located just rostral to the splenium of the corpus callosum; for the axial selection, the plane is located just below the level of the same structure. In the middle column the 2D fractional anisotropy color maps show the respective ROIs delineated (black line) within the white oval (added for easier identification). Using tracking algorithms that perform logical AND operands, the arcuate fibers are extracted. Third column shows a 3D slice with the arcuate tracts floating on top of it. These tracts are directional color coded: ascending fibers are blue to purple, fibers oriented posterior-anterior are cyan to green, and fibers directed right to left are red. Notice to both ends of the tracts the color is red. The rostral terminus in the left is red revealing the frontal-opercular connectivity, only present on the left side. Notice also the different orientation between 2D and 3D insets, the former with right on the left, the latter with left on the left side.

The fiber count (drawn streamlines) for each tract, provided automatically by the plugin, was annotated. Manually, the localization of the rostral terminus end-points was performed as defined in 
prior publication of our group [8]. The presence of rostral terminus end - points in the frontal operculum, that includes the inferior third of the pre - central gyrus (BA4 and BA6) and pars opercularis and triangularis of the inferior frontal gyrus (BA44, BA45) was determined by inspecting sagittal cuts conveniently placed at the operculum level. In addition, we ascertain the presence of Broca's end - points (Broca's Branch) and annotated the percentage of fibers reaching this point (BA44/BA45).

\section{Lateralization index ROI definition (Language fMRI)}

Clusters of fMRI activation of all subjects were added and divided in two territories: Frontal and temporo-parietal. For each aggregated cluster we kept all voxels within two Standard Deviations (frequency of presence) accounting for outliers. Thus, two large aggregates were obtained representing expressive and receptive language areas. The contours of the images on the left side (the largest) were flipped following the sagittal midline over the right hemisphere to obtain mirror homotopic ROIs. The proportion of voxels activated on the left side with respect all the voxels activated is called the lateralization index (LI).

\section{LI calculations}

Language LI

For each patient, and for each domain (expressive, receptive) LI was calculated utilizing the ROIs obtained above. The following mathematical formula was used:

$$
L I_{\ldots:}=\frac{\sum V o x \#_{L-R O I}-\sum V o x \#_{R-R O I}}{\sum V o x \#_{L-R O I}+\sum V o x \#_{R-R O I}}(1)
$$

Where $\sum V o x \#_{L-R O I}$, for instance, is the total number of active voxels contained in the Left region of interest. LI with negative values are considered right sided lateralized; LI with positive values are considered left sided lateralized. For purposes of qualitative categorization values between -0.2 and +0.2 were considered as indicative of bilateral representation.

\section{Arcuate Fasciculus LI}

For this purpose we applied the same formula as explained above:

$$
L I_{F I B E R}=\frac{\sum \text { Fiber }_{L}-\sum \text { Fiber }_{R}}{\sum \text { Vox\# }_{L}+\sum \text { Fiber }_{R}}(2)
$$

In this case, $\sum_{\text {Fiber } H_{L}}$ refers to the total fiber count intersecting both ROIs on the left hemisphere, condition that we accept as fibers conforming the AF.

The following conventions were used for the qualitative classification of the AF' rostral terminus:
L: presence of terminus only in the left frontal operculum

R: presence of terminus only in the right frontal operculum

B: presence of terminus in both of them

Broca's Branch presence, that is the presence of fibers reaching specifically BA44-BA45 in the pars opercularis and triangularis of the inferior frontal gyrus, was simple determined with a (yes/no) and, if present, the percentage of fibers that reach those structures was found.

\section{Statistics}

Pearson correlation coefficient between language LI and arcuate fasciculus LI was performed. A correlation coefficient of 0.3 was accepted as significant. Interaction between age, gender, IQ scores and each of the dependent variables listed in table 2, were explored to discard confounding effects utilizing ANOVA.

Data availability

Anonymized data will be shared by request of any qualified investigator.

\section{Results}

General results are presented in table 1. It is observed that language is lateralized to the left hemisphere in 27 participants, to the right in two, and bilaterally represented in the remaining four. The number of fibers (drawn streamlines) in the right and left AF, as well as the total number are quite variable, from 4 to 32 in the left $\mathrm{AF}$, and from 0 to 29 in the right. Similarly, the total number of fibers ranges from 8 to 61 . The AF LI is also variable; in eight cases is completely lateralized to the left $(\mathrm{LI}=1.0)$, and in one case there is not any lateralization (Index LI $=0.0$ ). In all cases AF fibers have a terminus in the left frontal operculum; 15 of which have also terminus in the right frontal operculum. 20 cases have fibers reaching the Broca area (see Broca branch). However, when there is a Broca branch only a percentage of the AF fibers, ranging from $5 \%$ to $100 \%$ (average $38.4 \%$ ) reaches the canonical Broca's area.

Table 2 presents the correlations between several of the measures analyzed. Only two of the correlations has a significance level: Negative correlation $(r=-0.817)$ between AF lateralization index and number of fibers conforming the right AF. There is also mild correlation $(r=0.316)$ between the number of fibers of both AF. The first finding is a marginal observation that most likely reflects the fact that the right AF fiber count shows much more variability than the left. The second is another marginal observation probably related to maturational factors. Strikingly, there is no association between main variables: Language fMRI and AF lateralization indices. 


\begin{tabular}{|c|c|c|c|c|c|c|c|c|c|c|c|c|}
\hline \multirow[t]{2}{*}{ Gender } & \multirow[t]{2}{*}{ Age } & \multirow[t]{2}{*}{$\begin{array}{l}\text { Verb } \\
\text { IQ }\end{array}$} & \multirow[t]{2}{*}{$\begin{array}{c}\text { Full } \\
\text { IQ }\end{array}$} & \multicolumn{2}{|c|}{$\begin{array}{l}\text { Language Laterality by } \\
\text { FMR }\end{array}$} & \multicolumn{4}{|c|}{ Arcuate connectivity } & \multirow[t]{2}{*}{$\begin{array}{c}\text { Frontal } \\
\text { Operculum }\end{array}$} & \multirow[t]{2}{*}{$\begin{array}{c}\text { Broca } \\
\text { branch }\end{array}$} & \multirow[t]{2}{*}{$\begin{array}{c}\text { Broca } \\
\text { branch } \\
\%\end{array}$} \\
\hline & & & & LI Expressive & LI Receptive & Lat & L-Count & R-Count & $\mathrm{Ll}$ & & & \\
\hline M & 17 & 91 & 86 & 0.99 & 0.98 & $\mathrm{~L}$ & 22 & 1 & 0.91 & $\mathrm{~L}$ & Yes & 54.5 \\
\hline $\mathrm{F}$ & 16 & 104 & 92 & 1 & 0.54 & $\mathrm{R}$ & 5 & 22 & -0.63 & B & No & \\
\hline $\mathrm{F}$ & 12 & & & 1 & 0.30 & B & 32 & 29 & 0.05 & B & No & \\
\hline M & 17 & & & 1 & 0.98 & $\mathrm{~L}$ & 16 & 2 & 0.78 & B & No & \\
\hline $\mathrm{F}$ & 18 & & & 0.56 & 0.58 & $\mathrm{~L}$ & 19 & 9 & 0.36 & B & No & \\
\hline $\mathrm{F}$ & 14 & 93 & 93 & 1 & 1 & $\mathrm{~L}$ & 26 & 18 & 0.18 & B & Yes & 100 \\
\hline $\mathrm{F}$ & 15 & 91 & 100 & 1 & 0.28 & $\mathrm{~L}$ & 13 & 0 & 1.00 & $\mathrm{~L}$ & No & \\
\hline $\mathrm{F}$ & 17 & & & -0.05 & 0.54 & $\mathrm{~L}$ & 21 & 8 & 0.45 & B & No & \\
\hline $\mathrm{F}$ & 19 & 89 & 86 & 1 & 0.40 & $\mathrm{~L}$ & 12 & 7 & 0.26 & B & No & \\
\hline $\mathrm{F}$ & 13 & 76 & 67 & 0.99 & 1 & $\mathrm{~L}$ & 5 & 3 & 0.25 & B & Yes & 20 \\
\hline $\mathrm{F}$ & 16 & 96 & 92 & 0.85 & 0.58 & $\mathrm{~L}$ & 10 & 3 & 0.54 & B & Yes & 10 \\
\hline $\mathrm{F}$ & 12 & 112 & 117 & 0.60 & 1 & $\mathrm{~L}$ & 13 & 0 & 1.00 & $\mathrm{~L}$ & Yes & 20.0 \\
\hline M & 21 & & & 1 & 0.32 & $\mathrm{~L}$ & 13 & 3 & 0.63 & $\mathrm{~L}$ & No & \\
\hline M & 17 & & & 0.46 & 0.54 & $\mathrm{R}$ & 6 & 16 & -0.45 & B & Yes (left) & 12.5 \\
\hline M & 12 & & & 1 & 0.69 & $\mathrm{~L}$ & 14 & 3 & 0.65 & $\mathrm{~L}$ & Yes & 57.1 \\
\hline $\mathrm{F}$ & 16 & & & 0.85 & 0.67 & $\mathrm{~L}$ & 20 & 9 & 0.38 & B & $\begin{array}{l}\text { Yes (Bi- } \\
\text { lateral) }\end{array}$ & 40 \\
\hline M & 17 & & & 0.92 & 0.97 & $\mathrm{~L}$ & 12 & 0 & 1.00 & $\mathrm{~L}$ & Yes & 41.6 \\
\hline M & 13 & 103 & 99 & 0.58 & 0.94 & $\mathrm{~L}$ & 17 & 4 & 0.62 & $\mathrm{~L}>\mathrm{R}$ & Yes & 53 \\
\hline $\mathrm{F}$ & 17 & 96 & 96 & 0.97 & 0.02 & $\mathrm{~B}$ & 26 & 29 & -0.05 & $\mathrm{~B}$ & Yes & 30.7 \\
\hline $\mathrm{F}$ & 16 & 99 & 93 & 0.42 & 0.83 & $\mathrm{~B}$ & 17 & 21 & -0.11 & $\mathrm{~B}$ & Yes & 35 \\
\hline $\mathrm{F}$ & 17 & 105 & 109 & 0.09 & 0.73 & $\mathrm{~L}$ & 12 & 0 & 1.00 & $\mathrm{~L}$ & Yes & 75 \\
\hline $\mathrm{F}$ & 15 & 106 & 101 & 1 & 1 & $\mathrm{~L}$ & 16 & 10 & 0.23 & B & Yes & 30 \\
\hline $\mathrm{F}$ & 19 & 77 & 103 & 1 & 0.65 & $\mathrm{~L}$ & 24 & 7 & 0.55 & $\mathrm{~L}>\mathrm{R}$ & No & \\
\hline $\mathrm{F}$ & 10 & 91 & 93 & 1 & 0.81 & $\mathrm{~L}$ & 24 & 12 & 0.33 & $\mathrm{~L}>\mathrm{R}$ & Yes & 45.8 \\
\hline $\mathrm{F}$ & 16 & 87 & 82 & 0.10 & 0.62 & $\mathrm{~L}$ & 13 & 0 & 1.00 & $\mathrm{~L}$ & No & \\
\hline $\mathrm{F}$ & 16 & 108 & 100 & 0.02 & 0.12954 & $\mathrm{~L}$ & 17 & 0 & 1.00 & $\mathrm{~L}$ & No & \\
\hline $\mathrm{F}$ & 23 & & 70 & 0.86 & 0.51 & $\mathrm{~L}$ & 13 & 4 & 0.53 & $\mathrm{~L}$ & No & \\
\hline $\mathrm{F}$ & 10 & 76 & 79 & 0.36 & 0.90 & B & 4 & 4 & 0.00 & L & Yes & 25 \\
\hline M & 12 & 90 & 78 & 0.64 & 0.99 & $\mathrm{~L}$ & 24 & 14 & 0.26 & B & Yes & 5 \\
\hline $\mathrm{F}$ & 10 & 118 & 127 & 0.99 & 1 & $\mathrm{~L}$ & 21 & 3 & 0.75 & $\mathrm{~L}$ & No & \\
\hline M & 13 & 102 & 92 & 1 & 0.87 & $\mathrm{~L}$ & 29 & 6 & 0.66 & $\mathrm{~L}$ & Yes & 17.2 \\
\hline M & 18 & 75 & 80 & -0.31 & 0.04 & $\mathrm{~L}$ & 27 & 0 & 1.00 & $\mathrm{~L}$ & Yes & 22.2 \\
\hline M & 15 & 112 & 111 & 1 & 0.63 & L & 20 & 0 & 1.00 & $\mathrm{~L}$ & Yes & 45 \\
\hline
\end{tabular}

Table 1: Description of the sample and MRI findings by participant.

Ll Expressive= Expressive language Lateralization Index. 1: completely left lateralized. -1 , completely right lateralized. Between -0.2 and 0.2 is considered bilateral; Ll Receptive= Receptive language Lateralization Index. Lat=Qualitative determination of laterality by comparing arcuate fasciculus thickness in tractography images; L- count=Automatic determination of Number of fibers conforming the LEFT AF (as defined fibers connecting Sup/Med temp gyrus with ipsilateral frontal operculum); R-count=Automatic determination of Number of fibers conforming the RIGHT AF (as defined fibers connecting Sup/Med temp gyrus with ipsilateral frontal operculum); Ll= AF index Technique to quantify lateralization: Any scalar on the left minus the scalar on the right divided by the sum of both; Frontal operculum=Presence of Left (L), right (R) or bilateral (B) rostral terminus in the frontal operculum (either Broca or premotor operculum); Broca branch= Presence or absence of fibers reaching canonical Broca's area, that is pars opercularis of the IFG; Broca branch\%= Percentage of fibers reaching canonical Broca, as compared to total fibers on the bundle. 


\begin{tabular}{|c|c|c|c|c|c|c|}
\hline \multicolumn{2}{|c|}{ Variable } & \multirow{2}{*}{$\begin{array}{c}\begin{array}{c}\text { Expressive } \\
\text { language } \\
\text { Lateralization } \\
\text { Index. }\end{array} \\
1\end{array}$} & \multirow{2}{*}{$\begin{array}{c}\begin{array}{c}\text { Receptive } \\
\text { language } \\
\text { Lateraliza- } \\
\text { tion Index. }\end{array} \\
.278\end{array}$} & \multirow{2}{*}{$\begin{array}{c}\begin{array}{c}\text { Number of } \\
\text { fibers left } \\
\text { Arcuate } \\
\text { fasciculus }\end{array} \\
.056 \\
\end{array}$} & \multirow{2}{*}{$\begin{array}{c}\begin{array}{c}\text { Number of } \\
\text { fibers right } \\
\text { Arcuate } \\
\text { fasciculus }\end{array} \\
.220\end{array}$} & \multirow{2}{*}{$\begin{array}{c}\begin{array}{c}\text { Arcuate } \\
\text { fasciculus } \\
\text { Lateraliza- } \\
\text { tion index }\end{array} \\
-.196\end{array}$} \\
\hline Expressive language & Pearson Correlation & & & & & \\
\hline Lateralization Index & $P=(2$-tailed $)$ & & .117 & .757 & .218 & .275 \\
\hline \multirow{2}{*}{$\begin{array}{l}\text { Receptive language } \\
\text { Lateralization Index }\end{array}$} & Pearson Correlation & & 1 & -.134 & -.189 & .030 \\
\hline & $P=(2$-tailed $)$ & & & .457 & .293 & .867 \\
\hline \multirow{2}{*}{$\begin{array}{l}\text { Number of fibers LEFT } \\
\text { Arcuate fasciculus }\end{array}$} & Pearson Correlation & & & 1 & .316 & .158 \\
\hline & $P=(2$-tailed $)$ & & & & .073 & .379 \\
\hline \multirow{2}{*}{$\begin{array}{l}\text { Number of fibers } \\
\text { RIGHT Arcuate fas- } \\
\text { ciculus }\end{array}$} & Pearson Correlation & & & & & $-.817^{* *}$ \\
\hline & $P=.(2-$ tailed $)$ & & & & & .000 \\
\hline $\begin{array}{l}\text { Arcuate fasciculus } \\
\text { Lateralization index }\end{array}$ & Pearson Correlation & & & & & 1 \\
\hline
\end{tabular}

Table 2: Correlation matrix.

Bold font: significant findings. (**): Strongest correlation obtained.

\section{Discussion}

Our results reveal significant variability in the representation of AF fibers and their lateralization. However, in all cases we noted a left frontal rostral terminus, either isolated or accompanied by similar connectivity in the right side (see Table 1, "Frontal Operculum"). In addition, in 20 of 33 subjects (61\%), AF fibers reached canonical Broca's area (pars opercular is) despite most fibers showing significant variability ( $5 \%$ to $100 \%$ ).

We additionally found no significant correlation between language LI (expressive or receptive) and either arcuate LI or frontal opercular lateralization (left or right) of its rostral terminus. These findings discourage the use of AF asymmetries to determine or increase the confidence of language lateralization.

\section{Concordant results}

Our results are concordant with studies conducted in normal adults undergoing language lateralization by fMRI and AF fiber density lateralization by DTI. In one study of 13 left handers, 5 evidenced right-sided language lateralization. However, all subjects had leftward asymmetry (80\%) or no asymmetry (LI < 0.10) [2]. Another study of 26 normal adult volunteers found discordant results between AF - related variables and language lateralization. $\mathrm{AF}$ variables studied included the bundle length and measures related to its reach and hence terminus [9]. A study of temporal - lobe - epilepsy patients correlated the lateralization of the AF fractional anisotropy and fMRI language lateralization in cases of right sided epilepsy [10].

\section{Discordant results}

A recent study of fMRI language and AF lateralization indices in 11 adult patients found a significant correlation $(R=0.739)$ when the AF LI was based on tract volume [11]. However opposite lateralization was found in one case. A study in normal children revealed a significant correlation between language lateralization by fMRI (verb generation and pair word matching paradigms) and volume-based AF LI ( $\mathrm{p}<0.02)$. However, this study included less than 10 cases [12]. Volume AF asymmetries have been found to correlate with language lateralization $(\mathrm{p}=0.02)$ in a study performed in 13 children with epilepsy [13]. However this study language lateralization was stablished by intracarotid amobarbital test, a test that in our experience is difficult and unreliable in young children. To our knowledge our study is the first to investigate this correlation with language fMRI in pediatric epilepsy patients.

Given the importance of the AF in language competence and its association with language processing [14-18], it is difficult to explain our discordant results. It is often assumed that the AF connects Wernicke's and Broca's areas and is directly involved in language repetition $[19,20]$. Its involvement in language competence is also suggested from comparative anatomy studies showing that the $\mathrm{AF}$ has a prominent temporal lobe projection in humans, but is much smaller or absent in nonhuman primates [21].

In the organization of brain fiber tracts the bundle anchor point or "terminus" suggests the specific function of the tract. For example, motor function can be inferred from ending points in primary 
motor cortex. The AF should therefore sub serve language functionality if at least one linguistic area is unequivocally connected. This network concept is supported by clinical studies inferring that the AF plays a fundamental role in language repetition as lesioning results in conductive aphasia (for a review see: Bernal and Ardila, 2009 [22]). Hence, it seems reasonable and theoretically acceptable, to correlate the degree of structural asymmetry with functional lateralization in right-handers [2].

Given this framework and the well-established anatomical asymmetry characterized by left hemisphere language dominance, it is striking to find negative results. It is possible that the $\mathrm{AF}$ has a transitory function in language learning that disappears with maturation. Performance in word learning has been correlated to the microstructural properties and strength of functional connectivity between Broca's and Wernicke's territories in the left hemisphere, suggesting that our ability to learn new words relies on efficient and rapid communication between the temporal and frontal areas [23]. Nevertheless this hypothesis seems feeble in front of recent findings in the trajectory of maturation of tracts associated to language functions. A study comparing the changes in fractional anisotropy, a marker of structural organization and maturation, of the aslant frontal fasciculi and the three segments of the superior longitudinal fasciculus (including the FA) evidenced increased fractional anisotropy associated to age, only occurring in the left $\mathrm{AF}$, in a group of children between 5 and 8 years of age [24]. It has been pointed, though, that the findings related to DTI processing may be influenced by the methods utilized in the acquisition or processing of fractional anisotropy and diffusivity [25]. There is paucity in the research of the effects of epilepsy on these biomarkers. There is a recent publication suggesting that functional connectivity abnormalities found in temporal lobe epilepsy have also representation in structural connectivity of the AF [26]. We did not factor this confound in our study. Anyhow, if the AF were required temporally for, say phonological learning, it should be mature enough at early ages.

Despite the widespread understanding that the AF always connects Wernicke's and Broca's areas, recent reports do not support this point of view. It has been reported that the AF projection to Broca's areas were absent in 10 out of 12 analyzed subjects $(83.3 \%)$, and minimal in $2(16.6 \%)$. However, the $\mathrm{AF}^{\prime}$ s rostral endpoints were found in the precentral gyrus in $100 \%$ of cases $[27,22]$. These findings were subsequently corroborated in a fiber dissection study of 12 post - mortem human hemispheres [28].

\section{Limitations and Problems}

It is possible that our results may be confounded by the effects of intractable epilepsy. Indeed, diffusivity coefficients of the AF has been found to increase in left and right temporal lobe epilepsy [29]. However, we believe this effect is unlikely to be significant because its presence should scramble the results. We observed the persistence of left lateralization even in cases of bilateral or right dominance.

Another potential limitation of our study is the lack of neuropsychological investigation in some cases. This is the result of conducting investigations in a clinical setting and utilizing retrospective data. However, we believe that all of our patients could understand and perform the controlled task suggesting that they had at least low average IQs.

The qualitative approach of determining the presence of anterior terminus within the frontal opercular cortex instead of a quantitative approach requires explanation. An alternative quantitative approach would need to measure maximal fiber length, as longer fibers would have a farther reach beyond the pre - central gyrus, up to the IFG. However AF fibers may be longer not because they reach the frontal operculum, but because the anterior terminus is located subcortically beyond the pre-central gyrus or because the posterior terminus extends below the middle temporal gyrus. Our method therefore guaranteed that the connecting endpoint was easily and accurately determined by visual inspection.

In summary, we have statistically compared the language lateralization provided by the arcuate fasciculus fiber count and language fMRI in a group of pediatric patients with intractable epilepsy. There was no significant correlation found between lateralization indices of the arcuate fasciculus and expressive or receptive lateralization indices obtained from activations on a controlled language fMRI. Our study discourages the usage of the AF lateralization as a biomarker of language lateralization in children with epilepsy. The findings are not necessarily applicable to normal populations or to patients with different conditions than intractable epilepsy, but concordant results seem to suggest the same direction.

\section{Financial Disclosure}

The authors state that they do not have any financial interest with regards data source, methods or results of this study. 


\section{Bibliography}

1. Nucifora PG., et al. "Leftward asymmetry in relative fiber density of the arcuate fasciculus". Neuroreport 16.8 (2005): 791794.

2. Vernooij MW., et al. "Fiber density asymmetry of the arcuate fasciculus in relation to functional hemispheric language lateralization in both right- and left-handed healthy subjects: a combined fMRI and DTI study". Neuroimage 35.3 (2007): 1064-1076.

3. Matsumoto R., et al. "Hemispheric asymmetry of the arcuate fasciculus: a preliminary diffusion tensor tractography study in patients with unilateral language dominance defined by Wada test". Journal of Neurology 255.11 (2008): 1703-1711.

4. Mandonnet E., et al. "Does the left inferior longitudinal fasciculus play a role in language? A brain stimulation study". Brain Journal of Neurology 130.3 (2007): 623-629.

5. Takao H., et al. "White matter microstructure asymmetry: effects of volume asymmetry on fractional anisotropy asymmetry". Neuroscience 231 (2013): 1-12.

6. Takao H., et al. "Gray and white matter asymmetries in healthy individuals aged 21-29 years: a voxel-based morphometry and diffusion tensor imaging study". Human Brain Mapping 32.10 (2011): 1762-1773.

7. Masutani Y., et al. "MR diffusion tensor imaging: recent advance and new techniques for diffusion tensor visualization". European Journal of Radiology 46.1 (2003): 53-66.

8. Bernal B and Altman N. "The connectivity of the superior longitudinal fasciculus: a tractography DTI study". Magnetic Resonance Imaging 28.2 (2010): 217-225.

9. Propper RE., et al. "A combined fMRI and DTI examination of functional language lateralization and arcuate fasciculus structure: Effects of degree versus direction of hand preference". Brain and Cognition 73.2 (2010): 85-92.

10. Rodrigo S., et al. "Language lateralization in temporal lobe epilepsy using functional MRI and probabilistic tractography". Epilepsia 49.8 (2008): 1367-1376.

11. Silva G and Citterio A. "Hemispheric asymmetries in dorsal language pathway white-matter tracts: A magnetic resonance imaging tractography and functional magnetic resonance imaging study". Neuroradiology Journal 30.5 (2017): 470-476.

12. Sreedharan RM., et al. "Arcuate fasciculus laterality by diffusion tensor imaging correlates with language laterality by functional MRI in preadolescent children". Neuroradiology 57.3 (2015): 291-297.
13. Tiwari VN., et al. "A sensitive diffusion tensor imaging quantification method to detect language laterality in children: correlation with the Wada test". Journal of Child Neurology 26.12 (2011): 1516-1521.

14. Dick AS and Tremblay P. "Beyond the arcuate fasciculus: consensus and controversy in the connectional anatomy of language". Brain Jouranl of Neurology 135.12 (2012): 3529-3550.

15. Mori S., et al. "Imaging cortical association tracts in the human brain using diffusion-tensor-based axonal tracking". Magnetic Resonance in Medicine 47.2 (2002): 215-223.

16. Catani $\mathrm{M}$ and Mesulam M. "The arcuate fasciculus and the disconnection theme in language and aphasia: history and current state". Cortex A Journal Devoted to The Study of the Nervous System and Behavior 44 (2008): 953-961.

17. Xia Y., et al. "Knowledge-based classification of neuronal fibers in entire brain". Med Image Comput Comput-Assist Interv MICCAI Int Conf Med Image Comput Comput-Assist Interv 8.1 (2005): 205-212.

18. Catani M and Thiebaut de Schotten M. "A diffusion tensor imaging tractography atlas for virtual in vivo dissections". Cortex $A$ Journal Devoted to The Study of the Nervous System and Behavior 44.8 (2008):1105-1132.

19. Damasio H and Damasio AR. "The anatomical basis of conduction aphasia”. Brain Journal of Neurology 103.2 (1980): 337350.

20. Geschwind N. "The clinical syndromes of the cortical connections". Modern Trends in Neurology 5 (1970): 29-40.

21. Rilling JK., et al. "The evolution of the arcuate fasciculus revealed with comparative DTI". Nature Neuroscience 11 (2008): 426-428.

22. Bernal B and Ardila A. "The role of the arcuate fasciculus in conduction aphasia". Brain 132.9 (2009): 2309-2316.

23. López-Barroso D., et al. "Word learning is mediated by the left arcuate fasciculus". Proceedings of the National Academy of Sciences of the United States of America 110.32 (2013): 1316813173.

24. Broce I., et al. "Fiber tracking of the frontal aslant tract and subcomponents of the arcuate fasciculus in 5-8-year-olds: Relation to speech and language function". Brain and Language 149 (2015): 66-76.

25. Wilkinson M., et al. "Detection and Growth Pattern of Arcuate Fasciculus from Newborn to Adult". Frontiers in Neuroscience 11 (2017): 389. 
26. Takaya S., et al. "Altered anterior-posterior connectivity through the arcuate fasciculus in temporal lobe epilepsy". $\mathrm{Hu}$ man Brain Mapping 37.12 (2016): 4425-4438.

27. Bernal B and Altman N. "The SLF does not connect to Broca's area”. 94th Sci. Assem. Annu. Meet. Radiol. Soc. N. Am (2008).

28. Martino J., et al. "Analysis of the subcomponents and cortical terminations of the perisylvian superior longitudinal fasciculus: a fiber dissection and DTI tractography study". Brain Structure and Function 218.1 (2013): 105-121.

29. Kim CH., et al. "Changes in language pathways in patients with temporal lobe epilepsy: diffusion tensor imaging analysis of the uncinate and arcuate fasciculi". World Neurosurgery 75.3-4 (2011): 509-516.

\section{Volume 2 Issue 10 October 2019}

(C) All rights are reserved by Byron Bernal., et al. 